\title{
Tuneable nanoparticle-nanofiber composite substrate for improved cellular adhesion
}

Ariana M. Nicolini ${ }^{a}$, Tyler D. Toth ${ }^{b}$, Jeong-Yeol Yoon ${ }^{a, b, c, *}$

a Biomedical Engineering Graduate Interdisciplinary Program, The University of Arizona,

Tucson, AZ 85721, USA

${ }^{b}$ Department of Biomedical Engineering, The University of Arizona, Tucson, AZ 85721, USA

${ }^{\mathrm{c}}$ Department of Agricultural and Biosystems Engineering, The University of Arizona, Tucson, AZ 85721, USA

* Corresponding author at: Department of Agricultural and Biosystems Engineering, The University of Arizona, 1177 E. 4th St., Tucson, AZ 85721-0038, USA. Tel.: +1 5206213587.

E-mail address: jyyoon@email.arizona.edu (J.-Y. Yoon).

(C) 2016. This manuscript version is made available under the Elsevier user license http://www.elsevier.com/open-access/userlicense/1.0/ 


\begin{abstract}
This work presents a novel technique using a reverse potential electrospinning mode for fabricating nanoparticle-embedded composites that can be tailored to represent various fiber diameters, surface morphologies, and functional groups necessary for improved cellular adhesion. Polycaprolactone (PCL) nanofibers were electrospun in both traditional positive (PP) and reverse potential (RP) electrical fields. The fibers were incorporated with $300 \mathrm{~nm}$ polystyrene (PS) fluorescent particles, which contained carboxyl, amine groups, and surfactants. In the unconventional RP, the charged colloidal particles and surfactants were shown to have an exaggerated effect on Taylor cone morphology and fiber diameter caused by the changes in charge density and surface tension of the bulk solution. The RP mode was shown to lead to a decrease in fiber diameter from $1200 \pm 100 \mathrm{~nm}$ (diameter \pm SE) for the nanofibers made with PCL alone to $440 \pm 80 \mathrm{~nm}$ with the incorporation of colloidal particles, compared to the PP mode ranging from $530 \pm 90 \mathrm{~nm}$ to $350 \pm 50 \mathrm{~nm}$, respectively. The nanoparticle-nanofiber composite substrates were cultured with human umbilical vein endothelial cells (HUVECs) and evaluated for cellular viability and adhesion for up to 5 days. Adhesion to the nanofibrous substrates was improved by $180 \pm 10 \%$ with the addition of carboxylated particles and by $480 \pm 60 \%$ with the functionalization of an RGD ligand compared to the PCL nanofibers. The novel approach of electrospinning in the RP mode with the addition of colloids in order to alter charge density and surface tension could be utilized towards many applications, one being implantable biomaterials and tissue engineered scaffolds as demonstrated in this work.
\end{abstract}

Keywords: reverse potential electrospinning; surface tension; surfactant; functionalized nanoparticles; RGD ligand. 


\section{Introduction}

Electrospun fibers of various materials and properties have been utilized in research such as tissue engineering, drug delivery systems, textiles, and biosensor applications [1]. According to Persano et al. [2,3], in the first decade of the $21^{\text {st }}$ century, the number of publications investigating properties and potential applications of electrospinning has increased exponentially. The volume of patents has also expanded at an astounding rate, and over $50 \%$ of these patents were related to biomedical scaffolds for use in implants. Electrospinning techniques are likely popular due to the formation of nano- and micrometer scaled structures that can be easily tailored to form smooth/rough, porous/non-porous, composite, 2D or 3D fibers, tasks unachievable through other filament-forming processes. Despite the volume of research to this end, there remain areas of electrospinning that require additional research and development, which will be discussed later in this paper.

Briefly, electrospinning is the process of creating fibers through the extraction of a solution - polymer:solvent - through a capillary tube while subjected to an electric field force. This field force drives the solution through an air interface where the solvent dissipates, thus resulting in ultrafine, polymeric fibers on a collection substrate. This event can only occur at a critical point in which the repulsive electrostatic forces at the surface of the polymer (i.e. airliquid interface) exceed the surface tension forces [4-6].

There are many methods that can be utilized to manipulate fiber diameter, surface chemistry, and topography during the electrospinning process. Such variables include applied voltage, tip-to-ground distance, inner needle diameter, extrusion rate, viscosity, volumetric charge density, and polymer:solvent ratio or type [6-8]. The addition of surfactants to polymer solutions has been a popular topic of research aimed at minimizing both beading during the 
spinning process as well as fiber diameter. By increasing the ratio of surfactant to polymer solution, the resulting fiber diameter can be estimated with respect to the forces on the Taylor cone and surface tension of the solution [9-11].

It is known that in certain circumstances micro- and nanometer solid particles can function similarly to surfactants and micelles [12]. Adsorption of particles and/or micelles to an air-liquid interface can affect surface tension $[13,14]$. This interaction occurs at a slower rate for colloids compared to that of individual surfactant molecules. However, for particles, adsorption is considered irreversible, whereas for surfactants, desorption occurs readily $[15,16]$.

To our knowledge, there has been no research to date that describes the use of particles for controlling surface tension and charge density during the electrospinning process. In addition to affecting surface tension and thus fiber morphologies, particles containing charged functional groups (carboxylated particles carrying negative charge and aminated particles carrying positive charge) could be integral in altering the electrostatic mobility of a nanofiber through the electric field during the electrospinning process. Reversing the potential of the electric field, i.e. from a positive applied voltage to the needle tip and grounded collection plate, vs. a grounded needle tip and positive collector voltage (Fig. 1), could lead to significant changes in fiber diameter [17,18], particle radial position within a fiber, distance between particles, and residual charges on the fibrous substrate. In addition to affecting surface topography and fiber diameter, the inclusion of functional particles allows for the coupling of biologically relevant peptides or ligands.

One possible application of nanoparticle-nanofiber composites is the formation of tissueengineered substrates that mimic the complex morphological structure of extracellular matrix (ECM). ECM is composed primarily of collagen fibrils ranging from $25-400 \mathrm{~nm}$ in diameter [19]. However, this diverse lattice can vary structurally, electrostatically, and biochemically 
depending on tissue function, location, and disease state. Topography, in particular random topography [20], and the presence of biochemical markers are crucial in cell-cell and cellsubstrate communication that promote focal adhesion, differentiation, migration, and proliferation [21-24].

In this work, we compare the relative effects of adding low concentrations of polystyrene particles $(300 \mathrm{~nm})$ into polycaprolactone electrospun nanofibers. We address the advantages and disadvantages of using both positive $(+12.5 \mathrm{kV})$ and reverse $(-12.5 \mathrm{kV})$ potentials for electrospinning with respect to: fiber diameter and its distribution, stability of the Taylor cone, surface tension as a predictor for fiber size, and surface topography. In addition, we investigate the relative effects of these parameters on the adhesion and viability of human umbilical vein endothelial cells (HUVECs), with the functionalization of the cell binding ligand RGD (arginineglycine-aspartic acid).

\section{Results and discussion}

\subsection{Interfacial effects of nanoparticles and surfactants}

In order to address the effects of the addition of colloidal particles on electrospun nanofiber diameter, polycaprolactone (PCL) solutions were prepared with carboxylated, surfactant-free carboxylated, non-carboxylated, aminated, and GRGDSPK ligand-functionalized polystyrene (PS) particles. Surfactant Tween 20 , at a concentration of $0.1 \%$, was added by the manufacturer to all carboxylated particles, except for surfactant-free carboxylated PS particles, and a cationic surfactant was added by the manufacturer to the aminated particles. Bovine serum albumin (BSA) was added to the GRGDSPK-particles in lieu of surfactant. As a control, a polymer solution containing the same non-ionic surfactant (Tween 20) was prepared to a final 
concentration of $0.087 \mathrm{mM}(0.009 \% \mathrm{w} / \mathrm{v})$. It was found that the addition of both surfactant and particles had no significant impact on nanofiber diameter when electrospun in the positive potential (PP) mode (Fig. 2). When electrospinning was performed in a reverse potential (RP) mode, a significant decrease in average fiber diameter was observed with the addition of colloidal particles (Fig. 2A). Interestingly, it was found that the relative time the surfactant was suspended in the solvent, 1,1,1,3,3,3-hexafluoro-2-propanol (HFIP), greatly affected the diameter of the fiber in the RP mode (Fig. 2B).

Although the interaction between non-ionic surfactants and fluorinated alcohols such as HFIP is relatively unknown, we hypothesize that the concentration of Tween 20 used in this system is well above that of the critical micelle concentration (CMC; $0.049 \mathrm{mM}$ in water) [25]. While HFIP is considered polar, like water, but its surface tension is much lower than that of water and it is immiscible with long chain alkanes such as those found in the hydrophobic region of polysorbate surfactants. The CMC of Tween 20 in 10\% PCL:HFIP was experimentally calculated as approximately $0.008 \mathrm{mM}$ (Supplementary Figure S1), which is substantially lower than the concentration used in this study $(0.087 \mathrm{mM}=0.009 \% \mathrm{w} / \mathrm{v})$. In the experiments with Tween 20 diluted in HFIP with varying storage time in polypropylene centrifuge tubes, it is expected that adsorption and desorption from the storage vessel walls and the air-liquid interface will occur. Due to hydrophobic interactions, excess surfactant will form micelles and potential large polyplex aggregates in the bulk solution over an extended time period. For example, after 3 or more days, the aggregates were further propagated through the effects of vortexing the polymer-solvent-surfactant solution. The adsorption towards the air-liquid interface is a relatively irreversible process for larger hydrophobic molecules and colloids, such as the surfactant micelles and colloidal particles used in this study. 
The addition of particles, surfactant, or a combination of the two affects the surface tension $(\gamma)$ of the polymer solution, as well as other properties important in electrospinning, discussed later. It is commonly known that increasing concentrations of surfactant to an aqueous phase leads to decreases in $\gamma$. The process of micelle formation and movement towards the airinterface are time and concentration dependent. As examined in this work, with very low concentrations of surfactant - close to the CMC while still higher than that - time becomes an important variable in assessing surface tension changes caused by the presence of surfactant.

\subsection{Surface tension and charge density in jet formation}

There are a variety of parameters that affect jet formation in the electrospinning process. In a most basic sense, a jet is formed from an extrusion orifice (needle tip) when an electric field is applied between the extrusion point and the collector plate. During this process the liquid solution becomes temporarily charged [26], leading to electrostatic repulsion from the bulk medium. Hence, jet formation only occurs when the Coulombic force from the applied electric field and the electrostatic repulsion of the surface charges overcome the surface tension at the extrusion point [27]. The ratio of these two primary forces (electrostatic/Coulombic force and surface tension) dictate the morphology of the Taylor cone and the final diameter of the fibers collected.

Several models have been reported for predicting nanofiber diameter $[7,8,28,29]$. One such example posed by Thompson et al. [7] included 13 distinct variables that influence the diameter of electrospun fibers. Of these variables it was theorized that distance from the nozzle, initial orifice radius, relaxation time, viscosity and volumetric charge density were the primary 
contributing factors in final fiber cross-sectional radii. This work also reported that surface tension had minimal effects on jet evolution and thus final fiber diameter.

In the current study, tip-to-ground distance, extrusion orifice radius, and extrusion rate were held constant. Viscosity and relaxation time will be minimally affected by the addition of colloidal particles, but cannot be responsible for the variations in fiber diameter observed in RP mode. Thus, charge density of the bulk solution must be the major contributing factor in the differences in fiber diameter observed in this work.

Charge density is primarily dependent upon the electrical properties of the bulk solvent, specifically conductivity and dielectric permittivity; both of which are insignificant for the polymer alone $[7,8]$. However, the addition of charged colloids to the system will affect the conductivity and thus charge density of the solution. Although the data in Fig. 4 suggests a strong correlation between surface tension and fiber diameter, where surface tensions were shown to increase slightly over the incubation time of Tween 20 solutions (T20-3+ > T20-1 > T20-0). Similarly, the addition of surfactant increased the $\gamma(\mathrm{C}-\mathrm{S}>\mathrm{C}-\mathrm{SF})$, and the addition of carboxylated PS particles also increased the $\gamma(\mathrm{C}-\mathrm{S}$ and C-SF > PCL). The differences in $\gamma$ are statistically insignificant. It is likely that the small changes in $\gamma$ are a secondary indicator of a primary phenomenon that alters fiber diameter. This primary factor is most likely solution charge density, or charge density in combination with viscosity and relaxation time.

\subsection{Taylor cone and fiber size distribution}

While electrospinning in the PP mode, it was observed that two distinct fibers are formed; in this instance they will be known as the primary and auxiliary fibers (Fig. 3). When electrospinning in the RP mode, a single distinct fiber is formed. It is apparent when assessing 
the distribution of fiber diameters (Fig. 3) that the RP mode produces larger, but more consistent fibers compared to the PP mode, in which nearly $50 \%$ of the fibers are formed from auxiliary jets. In the RP mode, only $0-20 \%$ of the fibers are formed from auxiliary jets. The formation of an auxiliary jet is due to both instability in the Taylor cone and low surface tension inherent with HFIP $(\gamma=16.14 \mathrm{mN} / \mathrm{m})$. Multi-jet spinning often occurs when the applied voltage is greater than that necessary for single-jet mode (Fig. 3) [30,31]. In other words, the force applied by the electric field is much greater than that necessary to overcome the surface tension. Although this is a typical means of decreasing fiber diameter, excessive electrical fields can also lead to phenomenon known as "beading”, spraying, or bubble jetting. Therefore, utilizing the RP mode with the addition of colloids and/or surfactant offers an alternative means of obtaining equally small fibers without the formation of secondary fibers or the possibility of the previously mentioned phenomenon in the PP mode.

\subsection{Particle spacing and surface topography of nanofiber-particle composites}

The particles chosen for this study were fluorescent, thus allowing for the analysis of spacing within the nanofibers. As observed in Figure 4, a smaller fiber diameter generally correlates to a larger spacing between two particles within a single fiber. Therefore, by adjusting other parameters in the electrospinning process such as tip-to-collector distance, needle tip diameter, or applied voltage, one could readily adjust the spacing of particles within each fiber. Due to electrostatic repulsion between the negatively charged particles and the negatively polarized PCL, a majority of the particles were found on the nanofiber surfaces (Fig. 5C,D). Electrospinning with the addition of colloids results in a more uniform surface topography than 
when electrospinning with the "beading" method [32-34]. Optimized spacing of surface features allows for enhanced cellular responses [35], which will be discussed in the next section.

\subsection{Cellular adhesion and RGD functionalization}

Human umbilical vein endothelial cells (HUVECs) were grown on the nanofiber and nanoparticle-nanofiber composite substrates. Cells showed good viability for up to 5 days in culture (Fig. 6A,C) on each substrate. Under centrifugal conditions (mimicking flow- or shearinduced culture), however, the cell viability was significantly compromised, while the addition of particles greatly improved the cell viability over 5 days (Fig. 6B,D). It is known that cellular adhesion is improved with enhanced surface topography [20,23,24,36,37], especially under flow or shear-induced conditions. In addition, the coupling of RGD containing cell-binding ligands (GRGDSPK in this work), prior to electrospinning, proved to increase cellular adhesion twice as well as that of carboxylated nanoparticle/nanofiber composite without RGD, and four times compared to standard PCL fibers (Fig. 6). The nanoparticle-nanofiber composite substrate without RGD peptide still demonstrated a nearly $2 \mathrm{x}$ increase in adhesion compared to standard PCL fibers.

According to Tran et al. [35], cell adhesion, particularly in endothelial cells, can be optimized when RGD ligands are not only bound to surfaces, but also separated by a particular distance $(1-5 \mu \mathrm{m})$ equivalent to the spacing of RGD-specific integrin on the surface of the cell. Choi et al. [38] showed that covalent coupling of the RGD ligand directly to the surface of electrospun polyurethane nanofibers also greatly improved cellular adhesion and spreading. Coupling of the ligand to particles prior to electrospinning can greatly reduce the quantity of RGD required to cover a large area of fibers [38]. Particles incorporated into the fibrous substrate 
will also contain the ligand beyond the 2D surface, as shown in previous methods. Hence, cells will in turn be promoted to spread deep into the substrate (Fig. 6E). This event, coupled with the enhanced topography created by the protruding nanoparticles, creates a fibrous substratum that encourages lamellipodial spreading of the endothelial cells, a morphology that is preferred by this type of mammalian cell.

As mentioned, the typical diameter of collagen fibrils in vivo appears in the sub-400 nm range, similar to the findings presented in this work. However, in the instance of the RP, solutions containing plain PCL as well as PCL with surfactant-free particles have much larger filament diameters, whereas the cellular data (Fig. 6D) shows similar adhesion to that of the smaller PP fibers. Tong and Wang explained that there are potential advantages of electrospinning for use in tissue engineering in the RP, but few researchers have accomplished this to date [39]. During the electrospinning process, excess charge can be accumulated on the fiber surface [26,40]. This phenomenon can remain up to 3 months [41]. It has been reported that surfaces containing net positive charges will promote improved cellular responses; this theory is particularly relevant in the presence of amine groups [42]. Amine-containing fibrous mats showed better adhesion within the first day, but after 5 days, adhesion decreased while the negatively charged carboxylated particles showed an overall increase in adhesion.

It is important to note that many added growth factors found in vivo and in vitro, such as vascular endothelial growth factor (VEGF) which has an isoelectric point of 8.5 [43], are positively charged (albeit weakly) in most cell culture media ( $\mathrm{pH} ~ 7.4)$. Others have shown that high concentrations of VEGF, 1-100 $\mu \mathrm{g}$ will adsorb towards PCL surfaces over long incubation times (> 24 hours) [44]. However in the unique RP mode where PCL is left with a net negative induced charge/polarity, it was hypothesized that adsorption towards these surfaces would occur 
more efficiently. One experiment was performed in order to quantify adsorption of VEGF toward the nanofibrous surfaces (Fig. 7). Surfaces were incubated at $37^{\circ} \mathrm{C}$ for 2 days with media (800 $\mu \mathrm{L}$ ) containing a final concentration of $4 \mathrm{ng}$ recombinant human (rh) VEGF per sample. Primary anti-VEGF conjugated with Alexafluor 647 was used for epi-fluorescence imaging. Increased signal in the red channel (Cy5 filter) was observed for the substrates electrospun in the RP mode (Fig. 7), thus indicating an increased concentration of VEGF at the surface. Although, VEGF adsorption is not truly necessary for cellular focal adhesion, presence of the growth factor will promote cellular proliferation.

\section{Conclusion}

Electrospinning in a reverse potential (RP) mode has seldom been studied in the past, likely due to an increase in fiber diameter, which is a disadvantageous trait for tissue engineered scaffolds. This work has shown that nanoparticles and surfactants incorporated into polymer solutions can lead to significant decreases in fiber diameters as well as an improvement in size consistency. Due to the fiber diameter distribution observed solely in the RP mode, the spacing of the functional particles could also be controlled. Integrin binding can be enhanced by optimizing particle spacing. In addition, the incorporation of functional particles into the nanofibrous substrates leads to improved endothelial cell adhesion under centrifugal sheer stress, an improvement caused by the complex surface topography of the nanoparticle-nanofiber composites and the availability of RGD ligands. The creation of nanoparticle-nanofiber composites are tailorable in morphology and functional properties as described in this work and have the potential to become useful in a variety of material applications outside of tissue engineered scaffolds. Such substrates could be useful in the future in a variety of platforms 
ranging from biosensing to smart fabrics to electrochemical sensors, all within the field of functional composite materials.

\section{Experimental section}

\subsection{Preparation of electrospun nanofiber/nanoparticle composites}

A $10 \% \mathrm{w} / \mathrm{v}$ solution was prepared by combining polycaprolactone (PCL, $80 \mathrm{kDa}$, SigmaAldrich, St. Louis, MO, USA) and 1,1,1,3,3,3-hexafluoro-2-propanol (HFIP, Sigma-Aldrich) for $2+\mathrm{hr}$ at room temperature on a rocking plate. $300 \mathrm{~nm}$ diameter yellow-green fluorescent polystyrene (PS) particles (final concentration of $0.25 \% \mathrm{w} / \mathrm{v}$ ) with varying surface functionality (carboxylated, non-carboxylated, GRGSDSPK conjugated, aminated, and surfactant-free; Magsphere, Pasadena, CA, USA) were separately added to the PCL solution. Surfactant:polymer solutions were prepared by diluting Tween 20 in HFIP to a concentration of $0.1 \%$. The $0.1 \%$ solution was added to the PCL:HFIP solution for a final surfactant concentration of $0.087 \mathrm{mM}$ $(0.009 \% \mathrm{w} / \mathrm{v})$. Each solution was vortexed for $2 \mathrm{~min}$ and was subsequently pumped through a 23 gauge blunt-tip Luer-Lock needle in a $1 \mathrm{~mL}$ syringe at a rate of $20 \mu \mathrm{L} / \mathrm{min}$ via a syringe pump. A DC voltage of $12.5 \mathrm{kV}$ (Gamma High Voltage Research, Ormond Beach, FL, USA) was applied to either the needle tip (positive potential) or the copper collection plate (reverse potential), and a ground was applied to the collection plate and needle tip, respectively. The tip-to-ground

distance was fixed at $12 \mathrm{~cm}$, and the entire electrospinning fixture was encased in a Plexiglas box to minimize external airflow. 


\subsection{Characterization of nanofibers}

Nanofibers were electrospun onto aluminum foil connected to the ground plate for analysis. After gold sputter coating for $60 \mathrm{~s}$, the samples were imaged using a Hitachi-S4800 field emission scanning electron microscope (SEM). Images were analyzed using ImageJ (National Institutes of Health, Bethesda, MA, USA). Surface tension was measured by the pendant droplet method via a surface tension/contact angle analyzer (FTÅ200, First Ten Ångstroms, Portsmouth, VA, USA). Droplets were extruded at a constant rate through an 18 gauge needle tip. Surface tension measurements were made prior to solvent evaporation at $15 \mathrm{~s}$. Taylor cone images were captured using the camera of FTÅ200.

\subsection{Functionalization of GRGDSKP peptide}

Covalent coupling of the RGD-containing peptide GRDGSPK (Anaspec Inc., Fremont, CA, USA) was performed using a modified protocol published by Invitrogen. Briefly, $56 \mu \mathrm{L}$ of peptide - for $3 \mathrm{x}$ coverage on the particle surface - was added to $30 \mu \mathrm{L}$ of $5 \mathrm{mM}$ MES buffer at $\mathrm{pH}$ of 6.0 , and $16 \mu \mathrm{L}$ of $2.5 \% \mathrm{w} / \mathrm{v}$ carboxylated particles (yellow-green fluorescent, $300 \mathrm{~nm}$; Magsphere). The solution was placed on a rocker plate for $15 \mathrm{~min}$, after which $160 \mu \mathrm{L}$ of 1 $\mathrm{mg} / \mathrm{mL}$ 1-ethyl-3-(3-dimethylaminopropyl) carbodiimide (EDAC) was added to the solution, and the $\mathrm{pH}$ was adjusted to 6.5. The solution was then placed on the orbital rocker for $2 \mathrm{hr}$ at room temperature. Finally, glycine was added to achieve a final concentration of $100 \mathrm{mM}$, and the reaction was quenched for $30 \mathrm{~min}$. After washing, the conjugated particles were suspended in 16 $\mu \mathrm{L}$ to achieve a theoretical concentration of $2.5 \% \mathrm{w} / \mathrm{v}$. 


\subsection{In vitro cell culture}

Primary human umbilical vein endothelial cells (HUVEC) were cultured in vascular cell basal medium, supplemented with an endothelial cell growth kit-VEGF (ATCC, Manassas, VA, USA), containing $5 \mathrm{ng} / \mathrm{mL}$ each of vascular endothelial growth factor (VEGF), epidermal growth factor (EGF), fibroblast growth factor (FGF), $15 \mathrm{ng} / \mathrm{mL}$ of insulin-like growth factor (IGF), 10 $\mathrm{mM}$ L-glutamine, $0.75 \mathrm{U} / \mathrm{mL}$ heparin sulfate, $1 \mu \mathrm{g} / \mathrm{mL}$ hydrocortisone, $50 \mu \mathrm{g} / \mathrm{mL}$ ascorbic acid, and $2 \%$ fetal bovine serum (FBS). Cultures were maintained at $37^{\circ} \mathrm{C}$ in a humidified $5 \% \mathrm{CO}_{2}$ incubator and passaged with 1x trypsin/EDTA (ATCC) at 80-90\% confluence, up to eight passages. Prior to substrate seeding, cells were resuspended in media to yield a concentration of 500,000 cells $/ \mathrm{mL} .50 \mu \mathrm{L}$ of the cell suspension was pipetted within $4 \mathrm{~mm}$ diameter $\mathrm{x} 3 \mathrm{~mm}$ deep wells formed from 10:1 polydimethylsiloxane (PDMS), which was placed on the surface of electrospun fiber mats collected on $1 \mathrm{~cm}$ x $1 \mathrm{~cm}$ stainless steel (SS) chips. SS chips were placed in a 24 well plate and cells were allowed to adhere $20 \mathrm{~min}$ within the incubated environment, after which the PDMS mold were removed and $600 \mu \mathrm{L}$ of media was added to each well. Cells were then incubated $4 \mathrm{hr}, 1,3$, and 5 days, with media replaced every $24 \mathrm{hr}$.

\subsection{Cellular viability, adhesion, and spreading}

HUVEC viability was assessed using ReadyProbes ${ }^{\circledR}$ cell viability imaging kit (blue/red, Life Technologies, Eugene, OR, USA). One droplet each of NucBlue ${ }^{\circledR}$ Live reagent (Hoechst 33342) and propodium iodide was added to each chip containing well and allowed to incubate for 15 min. Substrates were mounted on coverslips using vectashield (Vector Laboratories, Southfield, MI, USA) and imaged using an inverted epiflouorescent Nikon TS100 microscope and a 10x objective. Chips were quickly placed on a custom rapid prototyped tray retrofitted to 
hold the substrates in a vertical position within a $15 \mathrm{~mL}$ centrifuge tube filled with media. The substrates were then centrifuged at 3,300 rpm (rounds per minute) for $15 \mathrm{~min}$. after which detached cells were washed away with 1x Dulbecco's phosphate buffered saline (dPBS), remounted and imaged a second time. Initial viability was calculated as the percentage live/dead cells prior to centrifugation, and adhesion was found as total cells pre/post-centrifugation. Analysis was performed using ImageJ. Longer term (>3 days) cell spreading was characterized using both immunostaining and scanning electron microscopy. For both cases, cells were initially fixed using 4\% para-formaldehyde for $15 \mathrm{~min}$. For immunohistochemistry, cells were permeated with a $0.1 \%$ Triton X, $0.05 \%$ Tween 20 buffer solution. Following a washing step, non-specific binding was blocked using $0.5 \%$ bovine serum albumin (Sigma-Aldrich). Cells were then incubated in $1 \mathrm{mg} / \mathrm{mL}$ anti-vinculin (Millipore) diluted 1:250 in 1x PBS for 60 min. Cells were then washed and incubated with FITC-conjugated mouse anti-IgG and TRITC-Phalloidin for 60 min. Cells were then mounted and imaged at 100x using epi-fluorescent microscopy. For SEM, cells were dehydrated using deionized water from 3:1 - 1:1 - 1:3 water:ethanol dilutions at 5 $\mathrm{min} / \mathrm{step}$. Then 100\% ethanol - 3:1 - 1:1 - 1:3 - 100\% hexamethyldisilazane (HMDS, SigmaAldrich) dilutions at $5 \mathrm{~min} / \mathrm{step}$. Cells were allowed to dry overnight preceding gold sputter coating for standard SEM imaging.

A single data point in adhesion and viability experiments was an average of three different experimental replicates, each collected from a different well on the 24-well plates. Each well contained one stainless steel substrate coated with one of the six PCL nanofibernanoparticle composite materials electrospun in either the PP or RP modes. One set of data was collected varying time (4 hours, 1 day, 3 days, and 5 days) from different experimental replicates, i.e., on four theoretically identical stainless steel substrates electrospun from the same 
polymer mixture. The four substrates were simultaneously seeded and cultured with the cells from the same passage cycle. Epi-fluorescent images were collected from each individual stainless steel substrate. All experiments were repeated in triplicate, each time using newly fabricated substrates on a different well, and varying passage number of cells. Standard error was calculated with $n=3$.

\section{Acknowledgements}

We would like to thank the University of Arizona's Keck facility for access to imaging equipment. 


\section{References}

[1] Z. Huang, Y. Zhang, M. Kotaki, S. Ramakrishna. Compos. Sci. Technol. 63 (2003) 22232253.

[2] L. Persano, A. Camposeo, C. Tekmen, D. Pisignano. Macromol. Mater. Eng. 298 (2003) 504-520.

[3] A. Greiner, J. H. Wendorff. Angew. Chem. Int. Ed. Engl.46 (2007) 5670-5703.

[4] N. Bhardwaj, S. Kundu. Biotechnol. Adv. 28 (2010) 325-347.

[5] H. Fong, D. Reneker. J. Polym. Sci. B 37 (1999) 3488-3493.

[6] J. Doshi, D. H. Reneker. Conf. Rec. IEEE (1993) 1698-1703.

[7] C. Thompson, G. Chase, A. Yarin, D. Reneker. Polymer 23 (2007) 6913-6922.

[8] S. Theron, E. Zussman, A. Yarin. Polymer 45 (2004) 2017-2030.

[9] G. Taylor. Proc. Royal Soc. London A 313 (1969) 453-475.

[10] S. Wang, J. He, L. Xu. Polym. Int. 57 (2008) 1079-1082.

[11] Y. Liu, Z.-F. Ren, S.-Q. Wang. Int. J. Nonlin. Sci. Num. 11 (2010) 625-630.

[12] B. Binks. Curr. Opin. Colloid Interface Sci. 7 (2002) 21-41.

[13] J. Anderson. Ann. Rev. Fluid Mech. 21 (1989) 61-99.

[14] S. Khaleduzzaman, I. Mahbubul, I. Shahrul, R. Saidur. Int. Commun. Heat Mass Transfer 49 (2013) 110114.

[15] R. Miller, P. Joos, V. Fainerman. Adv. Colloid Interface Sci. 49 (1994) 249-302.

[16] J. Eastoe, J. Dalton. Adv. Colloid Interface Sci. 85 (2000) 103-144.

[17] Y. Yang, Z. Jia, Q. Li. IEEE Trans. Dielectr. Electr. Insul. 13 (2006) 580-585.

[18] P. Supaphol, C. Mit-uppatham, M. Nithitanakul. Macro. Mat. Engr. 290 (2005) 933-942.

[19] K. Gelse, E. Pöschl, T. Aigner. Adv. Drug Delivery Rev. 55 (2003) 1531-1546. 
[20] A. S. Curtis, B. Casey, J. O. Gallagher, D. Pasqui, M. A. Wood, C. D. Wilkinson. Biophys. Chem. 94 (2001) 275-283.

[21] R. O. Hynes. Cell 69 (1992) 11-25.

[22] A. Woods, J. R. Couchman. Collagen Rel. Res. 8 (1988) 155-182.

[23] N. Karuri, S. Liliensiek, A. Teixeira, G. Abrams, S. Campbell, P. Nealey, C. Murphy. J. Cell. Sci. 117 (2004) 3153-3164.

[24] C. Bettinger, R. Langer, J. Borenstein. Angew. Chem. Int. Ed. Engl. 48 (2009) 54065415.

[25] L. Wan, P. Lee. Pharm. Sci. 63 (1974) 136-137.

[26] G. Zaikov, E. Gennady, A. Valente, A. Iordanskii. Advances in Kinetics and Mechanism of Chemical Reactions (2013), Apple Academic Press: Toronto.

[27] K. Garg, G. Bowlin. Biomicrofluidics 5 (2011) 1-19.

[28] M. Hohman, M. Shin, G. Rutledge, M. Brenner. Phys. Fluids 13 (2001) 2201-2220.

[29] J. Feng. Phys. Fluids 14 (2002) 3912-3926.

[30] M. Cloupeau, B. Prunet-Foch. J. Electrostat. 22 (1989) 135-159.

[31] M. Enayati, Z. Ahmad, E. Stride, M. Edirisinghe. J. Royal Soc. Interface (2010) S393S402.

[32] Y. Liu, J. He, J. Yu, H. Zeng. Polym. Int. 57 (2008) 632-636.

[33] Y. Liu, L. Dong, J. Fan, R. Wang, J. Yu. J. Appl. Polym. Sci. 120 (2011) 592-598.

[34] N. Nagiah, U. Sivagnanam, R. Mohan, N. Srinivasan, P. Sehgal. Adv. Eng. Mater. 14 (2012) B138-B148.

[35] P. L. Tran, J. R. Gamboa, K. E. McCracken, M. R. Riley, M. J. Slepian, J. Y. Yoon. Adv. Healthc. Mater. 2 (2013) 1019-1027. 
[36] D. Miller, A. Thapa, K. Haberstroh, T. Webster. Biomaterials 25 (2004) 53-61.

[37] S. Biela, Y. Su, J. Spatz, R. Kemkemer. Acta Biomater. 5 (2009) 2460-2466.

[38] W. Choi, J. Bae, H. Lim, Y. Joung, J.-C. Park, I. Kwon, K. Park. Biomed. Mater. 3 (2008) 044104.

[39] H.-W. Tong, M. Wang. Biomed. Mater. 5 (2010) 054110.

[40] G. Collins, J. Federici, Y. Imura, L. H. Catalani. J. Appl. Phys. 111 (2012) 044701.

[41] H. L. Schreuder-Gibson, P. Gibson, P. Tsai, P. Gupta. INJ Winter (2004) 39-45.

[42] J. H. Lee, H. W. Jung, I. K. Kang, H. B. Lee. Biomaterials 15 (1994) 705-711.

[43] N. Ferrara, K. Houck, L. Jakeman, D.W. Leung. Endocr. Rev. 13 (1992) 18-32.

[44] S. Singh, B. Wu, J. Dunn. Biomaterials 38 (2011) 2059-2069.

[45] W. Preston. J. Phys. Chem. 52 (1948) 84-97. 


\section{Figure captions}

Graphical abstract. Tuneable electrospun nanofibers using polystyrene nanoparticles in reverse potential were demonstrated and tested for improved adhesion of endothelial cells under shearinduced conditions.

Figure 1. Schematic of the horizontal electrospinning setup in which positive and negative potentials can be applied (arrows) to the needle tip and collector plate. Particles in the polymer solution maintain a certain distance based on surface charge (A). Similar to surfactants, particles adsorb to the air-liquid interface, thus altering the solution surface tension and Taylor cone morphology (B).

Figure 2. (A) Average fiber diameters of positively and negatively electrospun $10 \% \mathrm{w} / \mathrm{v}$ PCLHFIP solution containing: $0.1 \% \mathrm{w} / \mathrm{v} 300 \mathrm{~nm}$ polystyrene carboxylated particles with (C-S) and without (C-SF) anionic surfactant, non-carboxylated particles with surfactant (NC-S), aminated particles with cationic surfactant (A-S), and GRGDSPK coupled carboxylated particles containing albumin (RGD). (B) Average fiber diameters with the addition of Tween 20 (T20) to HFIP solvent with incubation time of 0,1 , and $>3$ days All surfactant concentrations $=0.01 \%$ v/v. Error bars represent standard error $(n=5)$.

Figure 3. (A) Fiber diameter distribution becomes more uniform when electrospun in the reverse potential mode (indicate in red dashed lines). The positive potential mode leads to the formation of primary and auxiliary fibers. (B) When formed in the RP, the Taylor cone elongates due to the 
overall negative charge of the polymer. Less electrostatic forces are required for fiber formation in RP, thus leading to enhanced uniformity.

Figure 4. Surface tension measured via the pendant droplet method (inset) indicates a direct correlation to the average electrospun nanofiber diameter when produced in the reverse potential mode. The timing of Tween 20 introduction to the solvent dramatically changes the surface tension over the initial 3 days in solution (T20-0 to T20-3+). Error bars represents standard error $(\mathrm{n}=5, \mathrm{x}$-axis; $\mathrm{n}=3$, y-axis).

Figure 5. Intrafiber spacing of particles: (A) measured on overlaid fluorescent light images (B), depicted by blue arrow (scale bar $=20 \mu \mathrm{m}$ ). (C) SEM image showing position of particles on the surface of PCL fibers. (D) Particles are covalently conjugated with an RGD containing ligand which protrudes from the surface of the fibers in order for focal adhesion towards cells. (D) Integrin-RGD ligand interaction causes cell lamellipodia to reach for particle sites and intercalate with the fibers. Error bars represent standard error $(n=3)$. Statistical analysis was performed using a paired t-test, $*$ indicates $\mathrm{p}<0.05$, and $* * \mathrm{p}<0.01$.

Figure 6. Viability of HUVEC on positively (A) and negatively (C) electrospun PCLnanoparticle composite surfaces evaluated by Live/Dead staining. Centrifugal adhesion of HUVEC on positively (B) and negatively (D) spun nanofiber substrates cultured over 1, 3 and 5 days. Error bars represent standard error $(n=3)$. 
Figure 7. PCL nanofibrous mats were electrospun in the PP and RP modes. Media $(800 \mu \mathrm{L})$ containing rh VEGF (final concentration of $4 \mathrm{ng}$ per sample) was incubated at $37^{\circ} \mathrm{C}$ for 2 days. Substrates were immunostained using an Alexafluor 647 labeled primary anti-VEGF antibody. Bright field and Cy5 filtered imaging was performed on a Nikon C1si scanning confocal microscope (scale bars $=10 \mu \mathrm{m})$.

Supplementary Figure S1. Surface tension of 10\% w/v PCL in HFIP with the addition of Tween 20 was measured using the pendant droplet method. CMC can be estimated from the minimum surface tension value when plotted versus surfactant concentration [45]. 


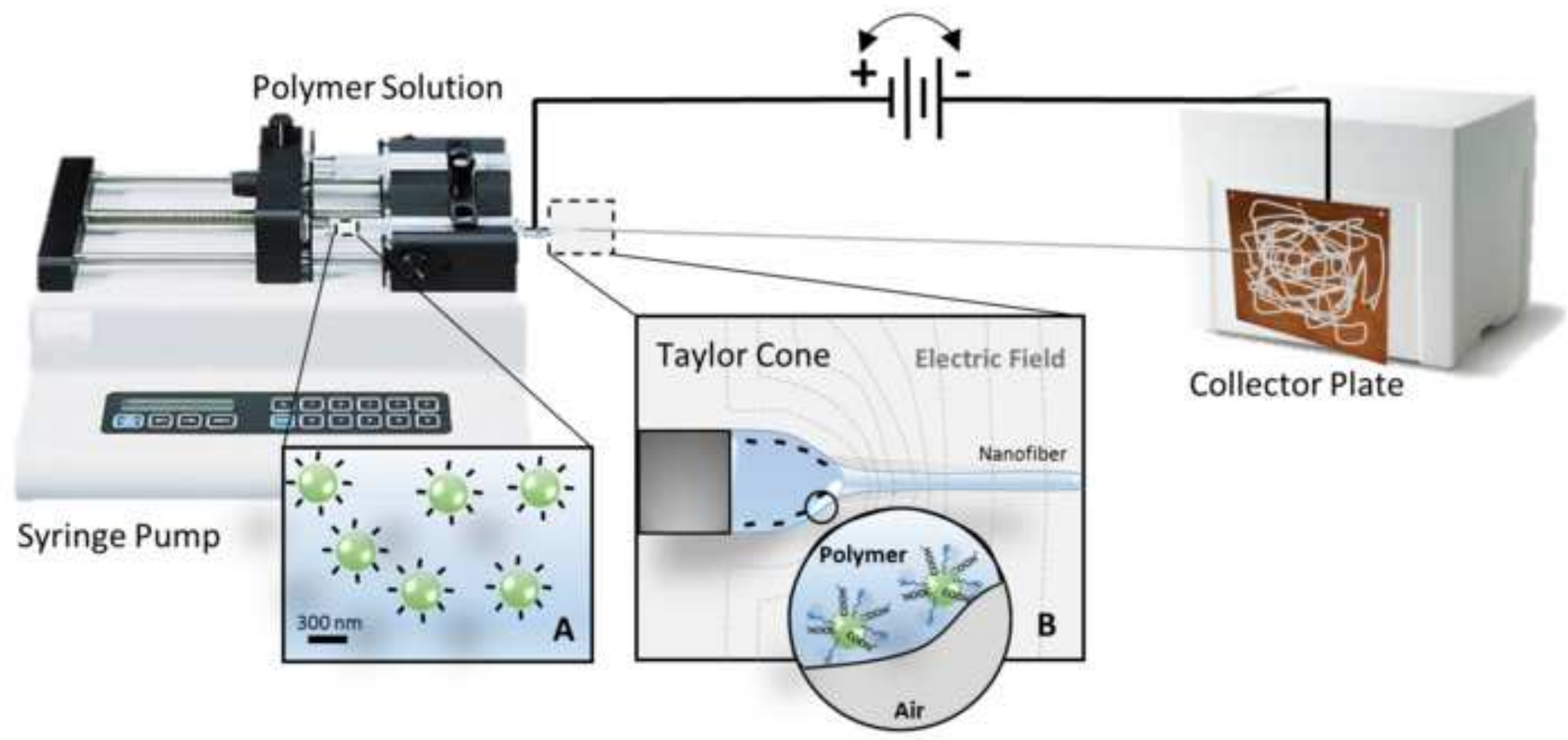




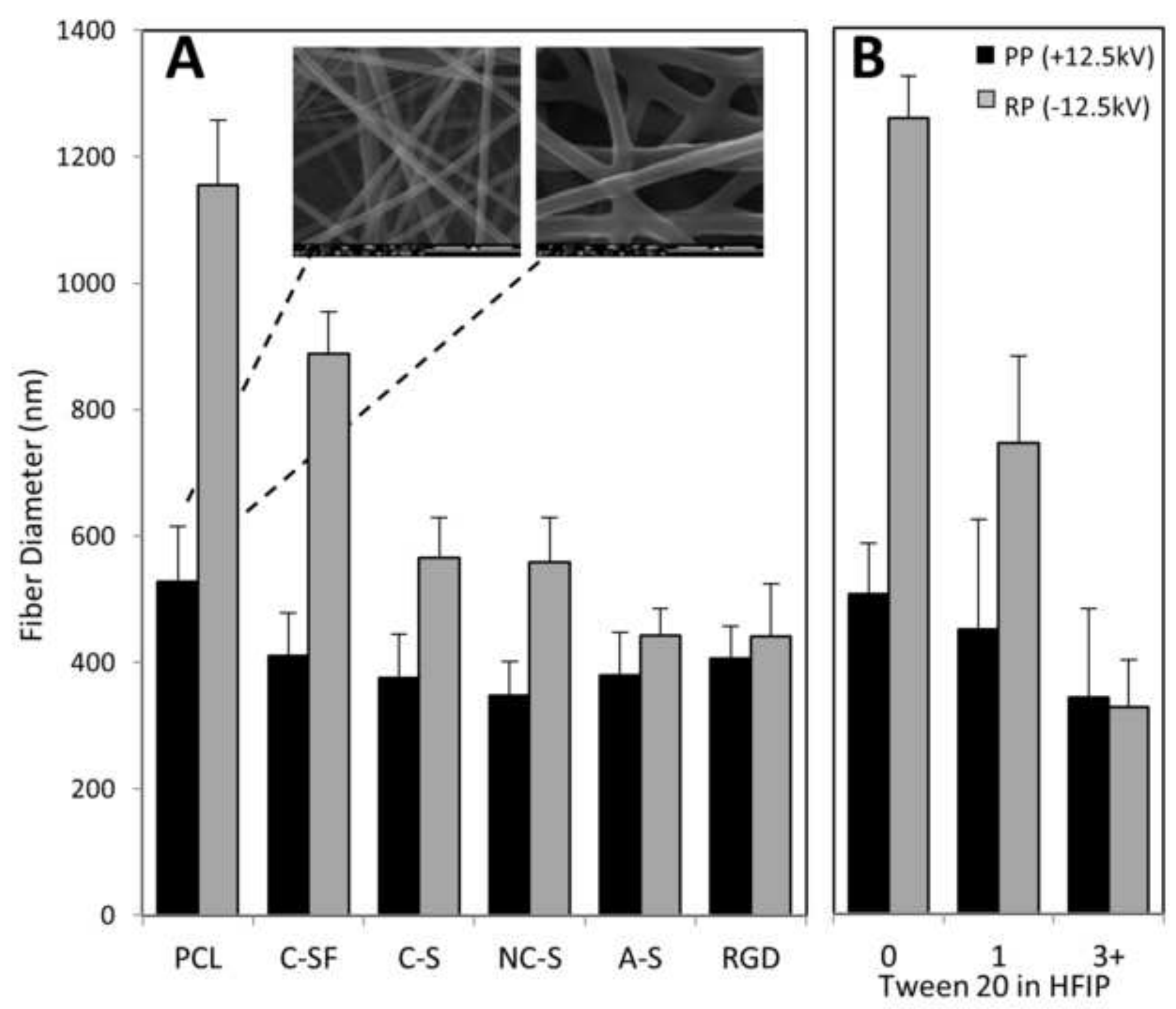



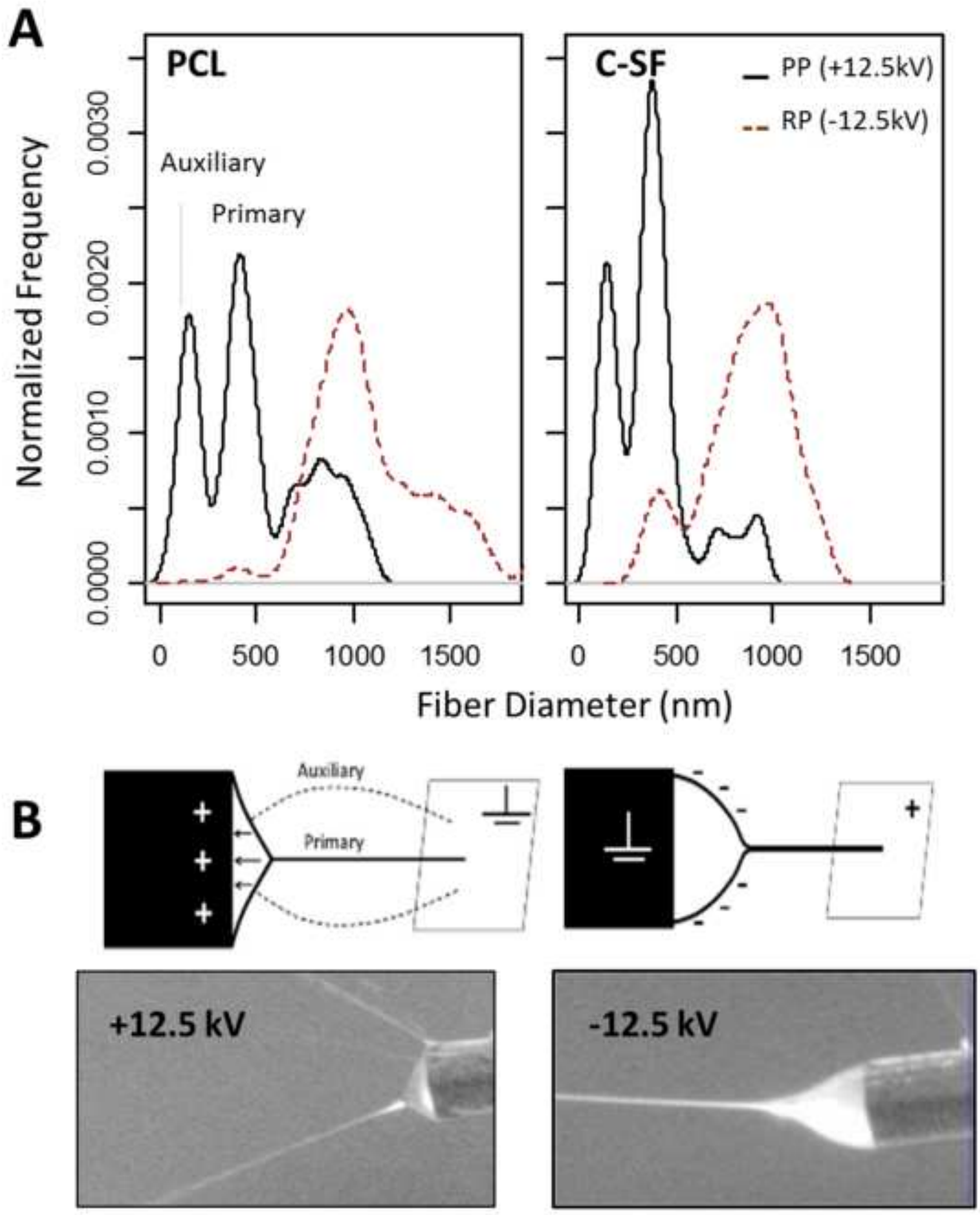


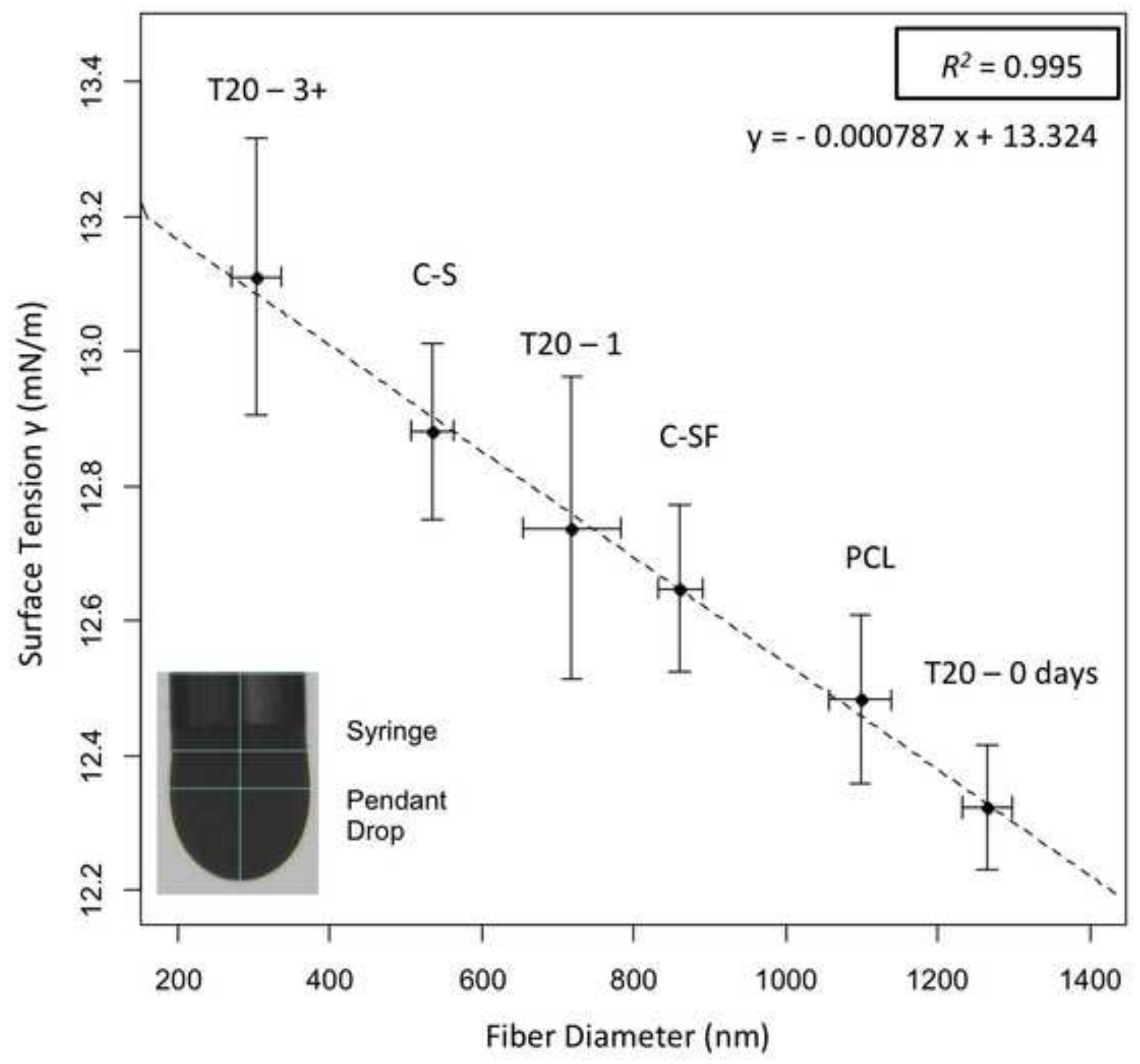



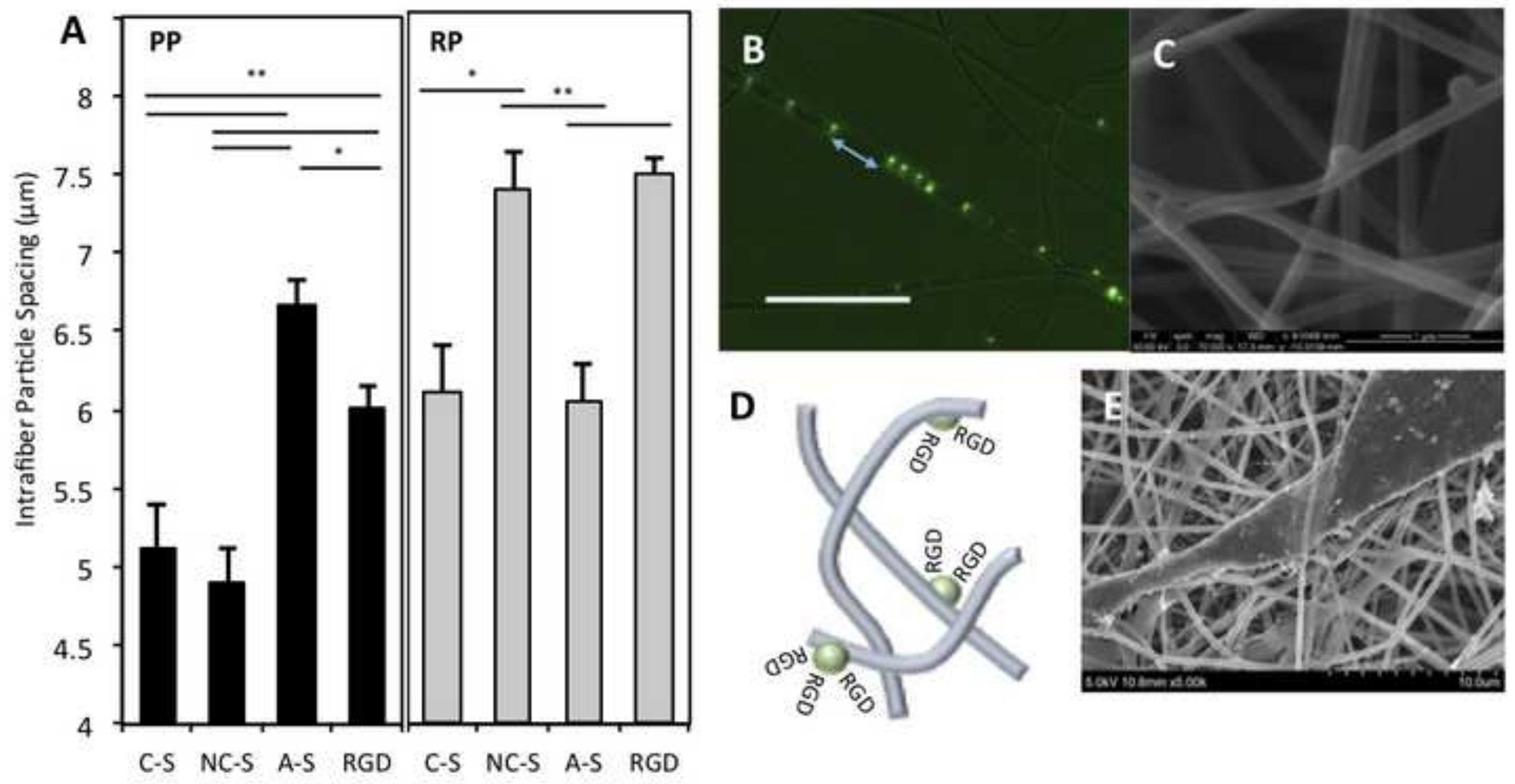

D
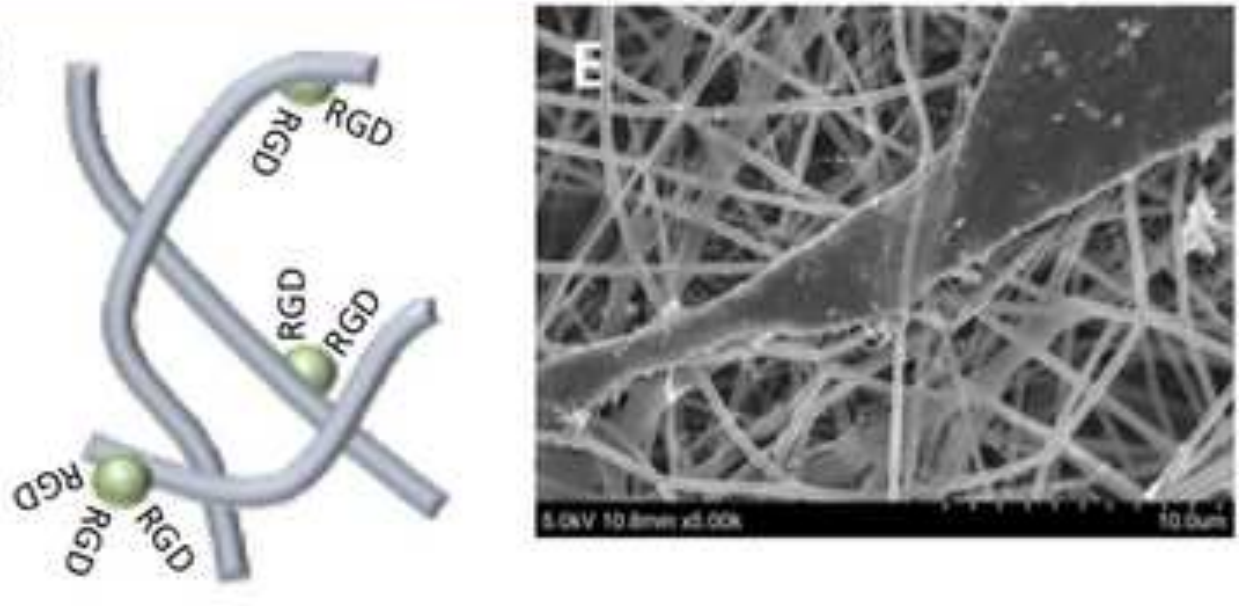

C-S NC-S A-S RGD C-S NC-S A-S RGD 


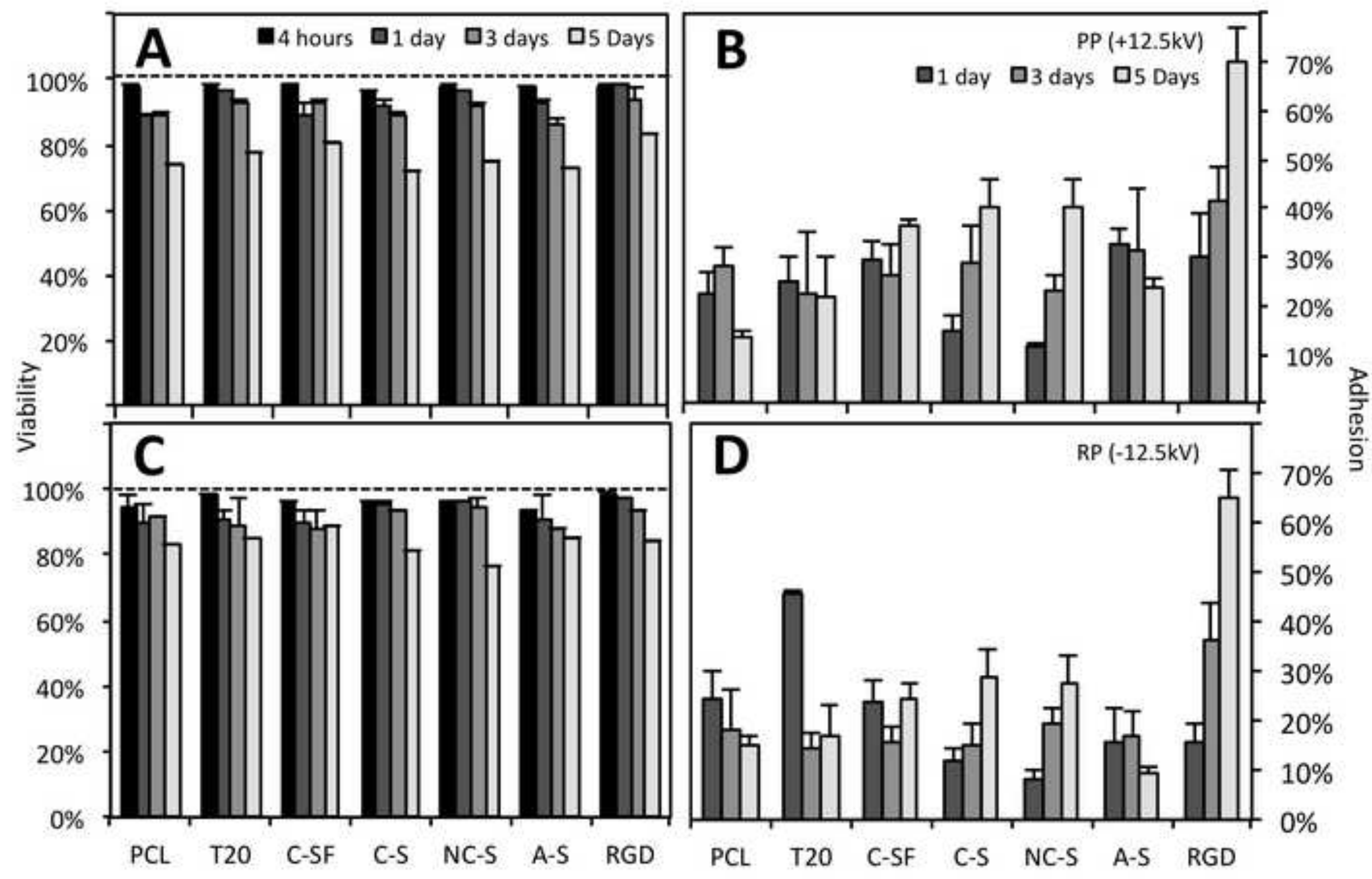




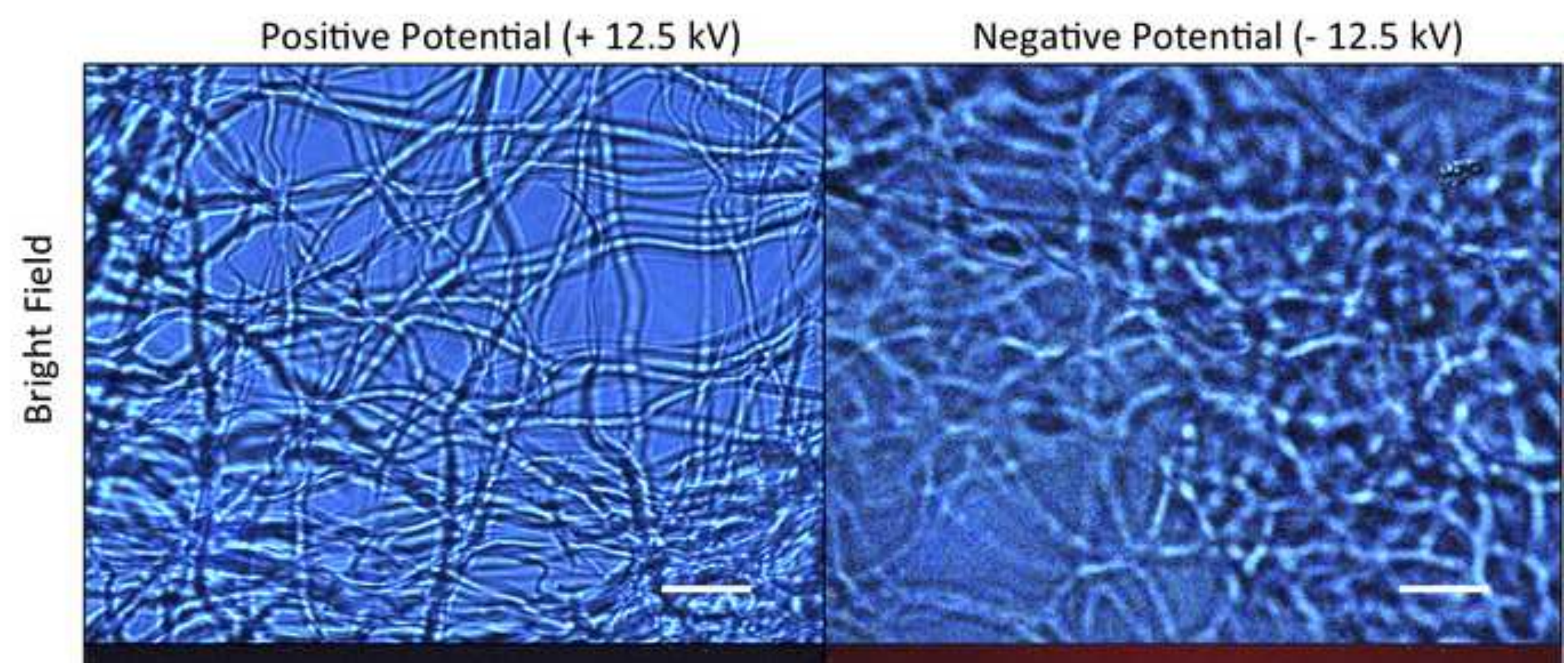

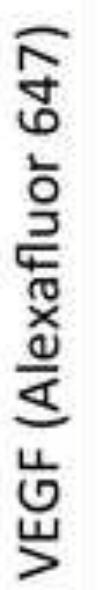

Positive Potential (+ 12.5 kV)

Negative Potential (- $12.5 \mathrm{kV})$ 




